\title{
A FORÇA DO TRABALHO FEMININO NA ODONTOLOGIA, EM ARAÇATUBA - SP
}

\author{
THE WOMEN WORKFORCE IN DENTISTRY IN ARAÇATUBA - SP
}

\author{
Suzely Adas Saliba MOIMAZ \\ Coordenadora do Programa de Pós-Graduação em Odontologia Preventiva e Social da Faculdade de Odontologia de Araçatuba - UNESP. \\ Nemre Adas SALIBA \\ Professora Titular do Departamento de Odontologia Infantil e Social da Faculdade de Odontologia de Araçatuba - UNESP. \\ Mikaela Reginee Basso BLANCO \\ Aluna do $4^{\circ}$ ano de Graduação da FOA - UNESP.
}

\begin{abstract}
$N$ śltimos anos tem havido um processo de feminilização nas profissões, principalmente na área da saúde.

Nesse trabalho, o objetivo foi analisar o exercício da odontologia por profissionais do sexo feminino, quanto à renda mensal obtida com a profissão, ao grau de satisfação e problemas enfrentados, dentre outros aspectos. Foram entrevistadas 100 cirurgiãs-dentistas, da cidade de Araçatuba, SP, utilizando-se um formulário com 30 perguntas. Os resultados mostraram que $87 \%$ disseram não ser a principal fonte de renda de sua família, $38 \%$ possuem renda mensal inferior à $\mathrm{R} \$ 1.500,00 ; 44 \%$ afirmaram que a renda obtida com o exercício profissional não é suficiente. Do total, $78 \%$ consideram-se satisfeitas com a profissão, porém 58,2\% não incentivariam seus filhos a cursarem odontologia. As principais queixas apontadas são: a baixa remuneração que a profissão lhes proporciona e a saturação do mercado de trabalho. Quanto à saúde, 50,5\% possuem algum problema relacionado à atuação profissional, principalmente dores na coluna e varizes. Conclui-se que embora a maioria das entrevistadas afirmou estar satisfeita com a profissão, os problemas financeiros, de saúde, as queixas e a não recomendação da profissão para o filho, traduzem a insatisfação, às vezes inconsciente, das mulheres no exercício da odontologia.
\end{abstract}

UNITERMOS: Trabalho feminino, odontologia; Saúde da mulher; Educação em odontologia.

\section{INTRODUÇÃO}

A participação da mulher como força de trabalho tem aumentado consideravelmente, nas diferentes atividades humanas. Na área odontológica esse fato tem sido observado e comprovado por diversos autores (Rosa, Madeira ${ }^{9}$ ). Em uma pesquisa realizada pela Universidade Federal Fluminense, verificou-se um aumento na demanda feminina nos cursos de Odontologia (Cornack $\left.{ }^{1}, 1988\right)$, o que também foi observado em um estudo realizado na Inglaterra (Matthews, Scully $\left.{ }^{4}, 1994\right)$ e outro pela Universidade Federal de Minas Gerais (Oliveira ${ }^{7}, 1983$ ).

Pesquisas sobre outros fatores relativos à prática profissional pelo sexo feminino demonstraram não haver discriminação contra as mulheres, porém discriminação não é o problema, serem tratadas exatamente nos mesmos termos que os homens o é, uma vez que tradicionalmente as mulheres assumem o compromisso de cuidar dos filhos e da casa, dificultando o exercício da profissão ( $\left.\mathrm{Du}^{2}, 1983\right)$, (Epson $^{3}$, 1974). O fator que mais afeta a carreira dessas profissionais são as crianças (McEwen, Seward5 , 1988), fazendo com que muitas optem por trabalhar somente meio período (Matthews, Scully ${ }^{4}$, 1994). Algumas das alternativas encontradas foram levar as crianças para o trabalho (Women ${ }^{14}$, 1975) ou deixá-las aos cuidados dos pais (Waldman ${ }^{13}, 1999$ ).

No estudo de Newton, et al. ${ }^{6}$ (2000) sobre o desenvolvimento da carreira de homens e mulheres na Odontologia dos Estados Unidos, verificou-se que as mulheres ocupavam posições mais baixas na hierarquia da Comunidade (Newton, et al. ${ }^{6}, 2000$ ). Outros autores relataram uma preferência do sexo feminino pelas áreas de Odontopediatria e Odontologia Social (Silveira, Córdon, ${ }^{11}$ 1984), possivelmente pelo fato da maior preocupação com a comunidade (Cornack, ${ }^{1}$ 1988) e também porque são mais gentis e delicadas para lidar com pacientes idosos e crianças (Du, ${ }^{2}$ 1983), (Stewart, Drummond $\left.{ }^{12}, 2000\right)$.

Diante das mudanças ocorridas nos últimos anos no mercado de trabalho, especificamente relativo à odontologia, os autores realizaram um estudo para avaliar o perfil das cirurgiãs-dentistas do município de Araçatuba, SP.

Os objetivos específicos foram conhecer, através de entrevistas a renda mensal estimada, o grau de satisfação e dificuldades na prática profissional. 


\section{MATERIAIS E MÉTODOS}

Após a aprovação do projeto pelo CEP (processo: 406/ 01), foi efetuada uma análise quanti-qualitativa, seguindose as normas estabelecidas para pesquisa com seres humanos, dos aspectos relacionados ao exercício da profissão por cirurgiãs-dentistas do município de Araçatuba, SP.

Foi elaborado um questionário pelos autores para ser utilizado na coleta de dados. Este questionário contendo tópicos sobre estado civil, número de filhos, época de formatura, renda obtida com a profissão, especialidade, formação de pós-graduação, local e condições de trabalho, dificuldades e grau de satisfação com a profissão; foi testado e aplicado na pesquisa.

Um estudo piloto, com 20 Cirurgiãs-dentistas, foi efetuado para testar o questionário e sanar as dúvidas para aplicação do mesmo.

Para a seleção das profissionais, foi solicitada à Vigilância Sanitária do município de Araçatuba, SP uma listagem com o nome e endereço das Cirurgiãs-dentistas. Foi também obtida uma listagem do CRO-SP. Após a conferência das listas, do total de 129 Cirugiãs-dentistas em exercício, concordaram em participar dessa pesquisa 100 profissionais, as quais foram entrevistadas.

Os dados coletados foram processados utilizando-se o Epi-Info V 6.02 e submetidos à análise estatística.

\section{RESULTADOS}

Os resultados relativos a esta pesquisa estão expressos nas Figuras de 1 a 3, referentes à época de formatura, tipo de pós-graduação e condição de trabalho no consultório. $\mathrm{O}$ Quadro 1 descreve as informações obtidas sobre a presença de dificuldades no início e ao longo da carreira, a compatibilidade entre a renda obtida com a profissão e as necessidades básicas, problemas de saúde relacionados à profissão, satisfação ao exercer a odontologia e o incentivo para o filho cursar odontologia. Já as Tabelas 1 e 2, apresentam a distribuição percentual das Cirurgiãs-dentistas em relação à época de formatura e renda, e os tipos de problemas relacionados à profissão respectivamente.

\section{DISCUSSÃO}

O processo de feminilização da força de trabalho na área odontológica é um achado constante nos dados bibliográficos (Rosa, Madeira, $\left.{ }^{9} 1980\right),\left(\right.$ Silva, Cruz,${ }^{10}$ 1996), (Mc Ewen, Seward, ${ }^{5}$ 1988). Rosa, Madeira ${ }^{9}$ (1980) observaram que houve no Brasil um incremento no número de mulheres na Odontologia, dado também constatado nos Estados Unidos e Europa (Mc Ewen, Seward ${ }^{5}, 1988$ ).

Acredita-se que uma das responsáveis pela tendência a feminilização da odontologia no Brasil seja a mudança na situação econômica que vem ocorrendo nas últimas décadas. Há tempos atrás, a força de trabalho feminina não era requerida na incrementação financeira da família, porém, com menores salários e mudanças culturais a situação vem se modificando (Rabello et $\mathrm{al}^{8}$., 2000).

Das 100 profissionais entrevistadas, verifica-se que $55 \%$ delas encontram-se casadas, e a maioria vivenciando a dupla jornada, ou seja, a rotina de cuidados com casa consultório, enquanto que $39 \%$ são solteiras, $5 \%$ divorciadas e $1 \%$ viúva, sendo que do total quarenta e quatro por cento afirmaram que a renda obtida não é compatível com suas necessidades básicas e $87 \%$ afirmaram não ser a principal fonte de renda da família. (Quadro 1). Entre as solteiras, 95\% dizem que a renda não é suficiente.

Nota-se, que grande parte destas permanecem sendo sustentadas pelos pais mesmo depois de formadas. Ao se verificar o ano de formatura, pode-se observar que $43 \%$ das entrevistadas formaram na década de 90. (Figura1)

Com relação ao número de filhos, $51 \%$ das profissionais

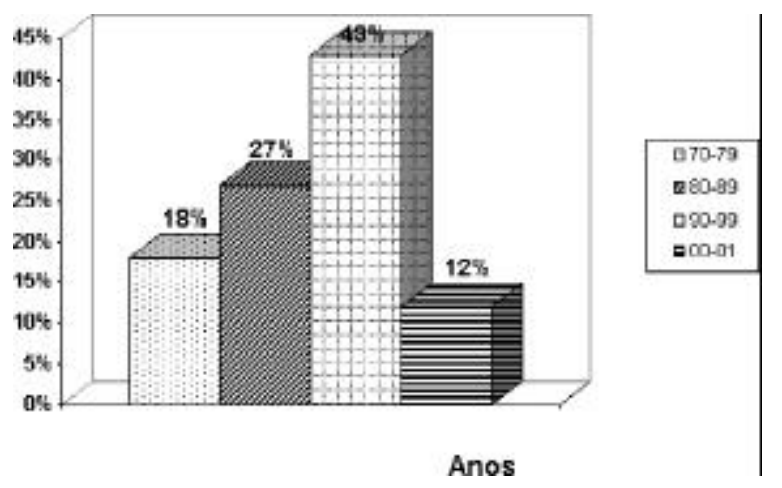

FIGURA 1- Distribuição das Cirurgiãs-dentistas, segundo a época de formatura. Araçatuba, SP-2002.

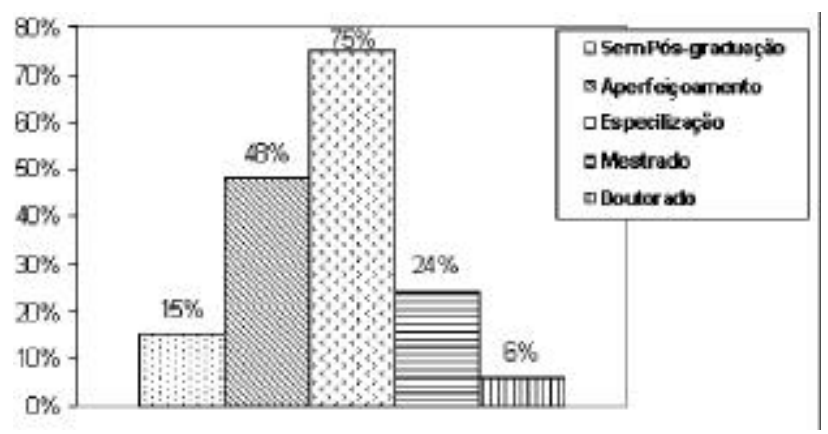

FIGURA 2- Distribuição das Cirurgiãs-dentistas, segundo o tipo de Pós-graduação cursado. Araçatuba, SP-2002.
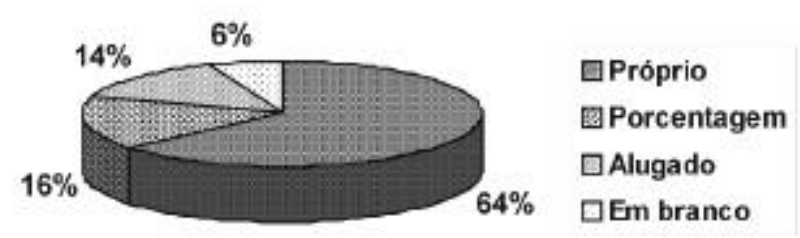

FIGURA 3- Distribuição das Cirurgiãs-dentistas entrevistadas, segundo a condição de trabalho no consultório. Araçatuba, SP-2002. 
QUADRO 1- Distribuição das Cirurgiãs-dentistas entrevistadas em Araçatuba (SP-2002), segundo os resultados sobre:

Presença de dificuldades no início da carreira

É a principal fonte de renda da família

Compatibilidade entre renda e necessidades básicas

Problemas de saúde relacionados à profissão

Presença de dificuldades ao longo do exercício profissional

Satisfação ao exercer a profissão

Incentivo para o filho cursar odontologia

\begin{tabular}{|cc|cc|cc|}
\hline \multicolumn{2}{|c|}{ Sim } & \multicolumn{2}{c|}{ Não } & \multicolumn{2}{c|}{ Total } \\
$\mathbf{n}$ & $\%$ & $\mathbf{n}$ & $\%$ & $\mathbf{n}$ & $\%$ \\
\hline 55 & 55,0 & 45 & 45,0 & 100 & 100 \\
13 & 13,0 & 87 & 87,0 & 100 & 100 \\
56 & 56,0 & 44 & 44,0 & 100 & 100 \\
50 & 50,5 & 49 & 49,5 & 99 & 100 \\
32 & 32,0 & 68 & 68,0 & 100 & 100 \\
78 & 78,0 & 22 & 22,0 & 100 & 100 \\
41 & 41,8 & 57 & 58,2 & 98 & 100 \\
\hline
\end{tabular}

TABELA 1- Distribuição percentual das Cirurgiãs-dentistas, em relação à época de formatura e renda. Araçatuba, SP-2002

\begin{tabular}{|c|c|c|c|c|c|}
\hline Década & 70 & 80 & 90 & 00 & Total \\
\hline Renda & $\%$ & $\%$ & $\%$ & $\%$ & $\%$ \\
\hline Menos de $R \$ 500,00$ & 0 & 0 & 1 & 3 & 4 \\
\hline De $R \$ 500,00$ até $R \$ 999,00$ & 1 & 1 & 10 & 4 & 16 \\
\hline De $R \$ 1000,00$ até $R \$ 1499,00$ & 1 & 2 & 12 & 3 & 18 \\
\hline De $R \$ 1500,00$ até $R \$ 1999,00$ & 3 & 4 & 8 & 1 & 16 \\
\hline De $R \$ 2000,00$ até $R \$ 2499,00$ & 0 & 4 & 5 & 0 & 9 \\
\hline De $R \$ 2500,00$ até $R \$ 3000,00$ & 2 & 3 & 4 & 0 & 9 \\
\hline Mais de $R \$ 3000,00$ & 11 & 13 & 3 & 1 & 28 \\
\hline Total & 18 & 27 & 33 & 12 & 100 \\
\hline
\end{tabular}

TABELA 2- Distribuição das 22 Cirurgiãs-dentistas insatisfeitas com a profissão, segundo os tipos de problemas relacionados à profissão. Araçatuba, SP-2002

\begin{tabular}{ll}
\hline Problemas & $\%$ \\
\hline Financeiro & $54,4 \%$ \\
Saturação do mercado de trabalho & $22,6 \%$ \\
Cirurgião-dentista não valorizado & $9,1 \%$ \\
Baixo poder aquisitivo do paciente & $4,5 \%$ \\
Falta de educação para a saúde bucal & $4,5 \%$ \\
Preconceito com o dentista recém-formado & $4,5 \%$ \\
Em branco & $0,4 \%$ \\
\hline
\end{tabular}

possuem um ou mais, e $37 \%$ delas afirmaram ter tido sua carga horária de trabalho diminuída após o nascimento deles. O fato de se diminuir a carga horária de trabalho também foi relatado pelos autores "Mathews, Scully (1994)".

Apenas $15 \%$ do total de entrevistados não realizaram qualquer curso de pós-graduação. A Especialização foi o curso de Pós-graduação de maior representatividade (75\%), seguida do Aperfeiçoamento, com $48 \%$ das profissionais. (Figura 2)

Dentre as que realizaram cursos de pós-graduação (85\%), verificou-se que as áreas de maior preferência são a Odontopediatria, com $42 \%$, depois a Cirurgia com $25 \%$ e a Dentística com $22 \%$, o que confirma os achados de "Silveira, Córdon (1984)". "Stewart, Drummond (2000)" e "Du (1983)" relataram que as mulheres preferem as áreas nas quais existe um maior campo de oportunidades para que elas possam propalar suas características tais como delicadeza, paciência e serviços que requerem habilidades manuais. Devido à necessidade de se buscar alternativas para o aumento da renda mensal, as profissionais atualmente partem para a realização de mais de uma especialização, tendo $32 \%$ das entrevistadas duas especialidades, sendo na maioria delas associada à Dentística ou Endodontia. Coincidentemente são as áreas relativas aos dois cursos existentes há mais tempo na Faculdade de Odontologia de Araçatuba - UNESP.

Referindo-se ao local de trabalho em que as entrevistadas atuam, verifica-se que $72 \%$ delas atendem somente em 
consultório, $12 \%$ em Consultório e Saúde Pública, $7 \%$ em Consultório e Instituição de Ensino, 5\% somente em Instituição de Ensino, 3\% em Saúde Pública e finalmente $1 \%$ em Instituição de Ensino e Saúde Pública. Das que trabalham somente em Consultório ou associado a outro local, $30 \%$ atuam em consultório alugado ou trabalham por porcentagem, 14\% e 16\%, respectivamente. (Figura 3)

Sendo inevitável a procura por trabalho, no mundo contemporâneo em que vivemos, a mulher tornou-se seletiva em relação à escolha de uma profissão que lhe possibilitasse uma maior flexibilidade de horários, permitindo-lhe a conciliação profissional e pessoal, o que de certa forma é oferecido pela odontologia.

Em relação aos motivos de escolha da profissão, a vocação foi apontada por $45 \%$ das entrevistadas como sendo o principal motivo de escolha, seguida da opção de se tornar um profissional "liberal" (40\%) e de terem tido algum tipo de contato com dentistas durante a infância ou adolescência (38\%). Este grande percentual referente à possibilidade de se tornar um profissional "liberal", na realidade diz respeito à possibilidade de se tornar um profissional autônomo, podendo assim administrar o seu próprio negócio. Observouse também que $55 \%$ das entrevistadas obtiveram alguma dificuldade no início da carreira, sendo a dificuldade financeira e insegurança que sente um profissional recémformado as de maior freqüência (Quadro 1). Outras dificuldades citadas foram à adaptação ao mercado de trabalho, a dupla jornada e o preconceito com profissionais recém-formados por parte dos pacientes.

Em relação à carga horária de atividade clínica e o número de dias em média trabalhados por semana, $65 \%$ delas afirmam trabalhar de 8 a 10 horas/diárias, em média cinco dias por semana $(60 \%)$.

Quanto à renda mensal, 20\% informaram receber menos de $\mathrm{R} \$ 1000,00,34 \%$ de $\mathrm{R} \$ 1000,00$ a $\mathrm{R} \$ 1999,00,18 \%$ de $\mathrm{R} \$ 2000,00$ a $\mathrm{R} \$ 3000,00$ e $28 \%$ obtém uma renda acima de R $\$ 3000,00$ mensais. Através do cruzamento dos dados da época de formatura e a renda mensal estimada, notou-se que dentre as profissionais formadas na década de 70, a maioria delas faturam acima de $\mathrm{R} \$ 3000,00$. Já as profissionais formadas nos últimos anos, possuem uma renda inferior quando comparada às outras, como mostra a Tabela 1, logo também, estas declararam que a renda obtida não é suficiente para suas necessidades. Do total de entrevistadas, $44 \%$ consideram que a renda obtida com a Odontologia não é compatível com suas necessidades.(Quadro 1)

Quase a totalidade das participantes (94\%) considerou que mantêm uma boa relação com colegas de profissão do sexo oposto e $50 \%$ consideraram ter obtido uma boa formação na faculdade, enquanto que $42 \%$ consideraram excelentes.

Quanto à saúde das profissionais entrevistadas, 50,5\% declararam possuir algum problema relacionado à atuação profissional, sendo com maior frequiência as dores na coluna e varizes. (Quadro 1)

O aumento do número de mulheres na profissão tem trazido conquistas e melhorias da posição da mulher na odontologia. Isto vem ocorrendo de forma que $68 \%$ não sentem dificuldade ao exercerem a profissão, $78 \%$ das profissionais consideram-se satisfeitas exercendo a profissão (Quadro 1). Dentre as $22 \%$ que não se sentem, 54,4\% têm como principal queixa a baixa remuneração que a profissão lhes proporciona, como mostra a Tabela 2. (Tabela 2)

Mesmo a maioria dizendo estar satisfeita com a profissão, 58,2\% dela não incentivaria seus filhos a cursarem odontologia. (Quadro 1)

\section{CONCLUSÃO}

A quantidade de especializações cursadas demonstra a preocupação com o sucesso profissional.

Quase metade das entrevistadas não obtém com a odontologia renda suficiente para satisfazer as suas necessidades.

Embora a maioria das entrevistadas afirmou estar satisfeita com a profissão, $57 \%$ delas não incentivariam seu filho a cursar odontologia. Este achado em conjunto com os problemas financeiros, de saúde e as queixas apontadas, traduzem a insatisfação, às vezes inconsciente das mulheres no exercício da odontologia.

Necessário se faz uma reflexão pelas instituições de ensino e entidades de classe sobre as conseqüências desse fato na prática odontológica.

\section{ABSTRACT}

A process of femininity has been recently occurring in the professions, mainly in the health field. In this study, the authors aimed at analyzing the dental practice by women as regards the income, degree of satisfaction and problems they have been facing. One hundred female dentists from Araçatuba, SP, were interviewed and answered a questionnaire designed for that purpose, which contained thirty questions. The results showed that $87 \%$ are not the main source of income of the family. Among the interviewed, $38 \%$ have an income below $\mathrm{R} \$ 1500 ; 44 \%$ agreed that the income from dental practice is not enough. From all, $78 \%$ considered themselves as satisfied with the profession, however $58.2 \%$ would not stimulate their children to pursue the dental career. The main complains are the low income and saturation of the profession. Concerning health, $50.5 \%$ have some professional diseases, mainly backache and varicose veins. It was concluded that, even though most subjects stated to be satisfied with the profession, aspects such as financial and health problems, complains and no recommendation of this career for their children demonstrate their sometimes unconscious dissatisfaction with the dental practice.

UNITERMS: Women working, dentistry; Women health; Dental education. 


\section{AGRADECIMENTOS}

Ao CNPq pela concessão de bolsa PIBIC e as Cirurgiãsdentistas que colaboraram com a participação nessa pesquisa.

\section{REFERÊNCIAS BIBLIOGRÁFICAS}

1- Cornack EF. O aumento da demanda feminina nos cursos de Odontologia:O caso da UFF. Rio de Janeiro; 1988. [Tese de Mestrado - Universidade Federal Fluminense].

2- Du TS. The women dental student-a report. Diastema 1983 11: 22-6.

3- Epson JR, Daniel N, Keller E. Report on part- time work for women dentists with family commitments. Br Dent J 1974; 136 (12): 513-4.

4- Matthews RW, Scully C. Work paterns of male and female dentists in the UK. Br Dent J 1994; 176(12): 463-6.

5- Mcewen EN., Seward MH. The Contribution of women to dentistry in the 1980s. Br Dent J 1988 nov; 165(9): 339-41.

6- Newton JT, Thorogood N, Gibbons DE. A study of the career development of male and female dental practitioners. Br Dent $\mathrm{J}$ 2000;188 (2): 90-4.

7- Oliveira $\mathrm{G}$ et al. O exercício profissional da Odontologia pela mulher grávida. Arq. Centro Estud. Odontol 1983; 20(1/2): 11527.

8- Rabello SB et al. Por que a Odontologia se transformou numa profissão de mulher? Rev Bras Odontol. Disponível em: <http:// www.odontologia.com.br/artigos.asp > Acesso em: 17/03/2001.

9- Rosa JE, Madeira AA. Participação da mulher na odontologia catarinense. Rev Catarinense Odontol. 1980;7(1): 19-25.

10-Silva EMC, Cruz ACS. Por que as mulheres escolhem a odontologia? Revista do CROMG 1996; 2(2): 65-9.

11-Silveira H, Córdon J. A mulher no ensino superior em odontologia no Brasil. Rev Fac Odontol. Porto Alegre 1984; 26 25-36.

12- Stewart FM, Drumond JR. Women and the world of dentistry. Br Dent J 2000; 188(1): 7-8.

13- Waldman HB. When mom is out working: dads to take care of the kids. ASDC J Dent Child 1999; 66(2): 140-4.

14- Women in Dentistry. Br Dent.J 1975; 139(4): 153-6.
Recebido para publicação em: 20/01/2003

Enviado para reformulações em: 19/03/2003

Pronto para publicação em: 26/06/2003

Endereço para correspondência:

Prof. Dra. Suzely Adas Saliba Moimaz

Faculdade de Odontologia de Araçatuba - FOA - UNESP.

Departamento de Odontologia Infantil e Social.

Rua José Bonifácio, 1193 - Caixa Postal 341

CEP-16015-050. Araçatuba - SP.

Fone: (18) 620-3250. e-mail: secrdos@ foa.unesp. Br 